\title{
Geometric Phase of an Open System
}

\author{
N. BuRIC* AND M. RADONJIC \\ Institute of Physics, Belgrade, Serbia
}

\begin{abstract}
Quantum state diffusion unraveling of the Linblad master equation is utilized to define a geometric phase of an open quantum system. It is then shown that such geometric phase is invariant under unitary symmetry transformations of the Linblad equation, which is important property not shared by the geometric phases based on other types of unraveling.
\end{abstract}

PACS numbers: 03.65.Vf

\section{Introduction}

State vectors $|\psi\rangle$ of a quantum system are normalized and the overall phase $\alpha$ in $\mathrm{e}^{\mathrm{i} \alpha}|\psi\rangle$ has no physical relevance. Nevertheless, two vectors on an orbit of the evolution governed by the Schrödinger equation can have nonzero relative phase $\alpha_{\text {tot }}=\arg \langle\psi(0) \mid \psi(t)\rangle$ which is measurable. It was Berry [1] who first realized that the total relative phase $\alpha_{\text {tot }}$ can be represented as a sum of the dynamical part that depends explicitly on the Hamiltonian and the part which is of a geometric origin. Berry considered evolution with Hamiltonian $\hat{H}(\boldsymbol{R}(t))$ depending on adiabatically and periodically changing parameters $\boldsymbol{R}$, and was able to show that a part of the total phase acquired by the systems state vector during one period of $\boldsymbol{R}(t)$ depends only on the geometric properties of the curve $\boldsymbol{R}(t)$ in the parameter space [1, 2]. It was soon realized that there is a whole class of such geometric phases that appear responsible for important physical effects [3]. In particular, the geometric phase was defined for curves in the space of pure states that did not rely on the adiabatic [4] or cyclic evolution [5,6]. It became clear that these geometric phases are always related to the geometry of the system evolution in the state space $\boldsymbol{P} H$, which has a nonzero curvature in the natural connection determined by the Hilbert space scalar product $[2,5,7]$. It is no surprise that the quantum information processing revolution brought the idea that the geometric phase can be used for quantum computing, which has been termed geometric or, more generally, holonomic computing (see for example [8]).

However, realistic quantum systems must be treated as open systems, i.e. together with their environment. The state of an open system is in general not pure and is described by a density matrix. Evolution of open quantum systems is necessarily described in terms of transformations of the density matrices even if the initial state is pure. There have been several attempts to define the ge-

* corresponding author; e-mail: buric@phy.bg.ac.rs ometric phase for mixed states of an open systems (see for example [9-15]). Most of them explore the representation of the mixed state of the open system in terms of a collection of pure states of the open system or in terms of reduction of a pure state of a larger isolated system. For example, the quantum jumps unraveling of the Linblad master equation for the evolution of the mixed state $\hat{\rho}(t)$, was used in [13] to define the geometric phase using the pure states that appear in the unraveling. However, often the stochastic dynamics of the pure states, that simulate the master equation for the mixed state, is not invariant under the transformations that are the symmetry of the mixed state master equation. Then the geometric phase defined using the pure states is not invariant under the symmetry transformations of the master equation, which cannot be considered as satisfactory. This fact was pointed out in [16]. However, there is a stochastic unraveling of the master equation in terms of pure state stochastic evolution, given by the quantum state diffusion (QSD) theory [17], which has the same symmetry as the master equation. The QSD stochastic evolution for pure states of the open system is uniquely defined given the mixed state master equation, and we shall show that it can be used to uniquely associate a geometric phase with the mixed state evolution.

\section{Geometric phase}

In the sequel we consider open quantum systems that satisfy Markov property. The most general continuous evolution of such a system is given by the GoriniKossakowski-Linblad master equation (LME) [18] for the density matrix $\hat{\rho}(t)$ :

$$
\begin{aligned}
& \frac{\mathrm{d} \hat{\rho}(t)}{\mathrm{d} t}=-\mathrm{i}[\hat{H}, \hat{\rho}] \\
& \quad+\sum_{m}\left(2 \hat{L}_{m} \hat{\rho} \hat{L}_{m}^{\dagger}-\hat{L}_{m}^{\dagger} \hat{L}_{m} \hat{\rho}-\hat{\rho} \hat{L}_{m}^{\dagger} \hat{L}_{m}\right),
\end{aligned}
$$

where $\hat{H}$ generates unitary evolution and the Linblad operators $\hat{L}_{m}$ describe the non-unitary influences of the environment. The LME (1) is invariant under the unitary transformations of the Linblad operators 


$$
\hat{L}_{m} \rightarrow \sum_{k} u_{m k} \hat{L}_{k}, \quad \sum_{m} u_{m k} u_{m k^{\prime}}^{*}=\delta_{k k^{\prime}},
$$

where $*$ denotes complex conjugation of the complex numbers $u_{m k}$. Consequently, an observable quantity related to an orbit $\hat{\rho}(t), t \in\left(t_{0}, t_{1}\right)$ must not depend on $u_{m k}$. However, it was pointed out in Ref. [16] that the definitions of the geometric phase based on stochastic unraveling of using particular stochastic Schrödinger equation with real Wiener noise or the quantum jumps approach are not invariant on the transformations of the type $\hat{L}_{m} \rightarrow \mathrm{e}^{\mathrm{i} \alpha} \hat{L}_{m}$ which are the symmetries of LME.

The idea to analyze the deterministic mixed state evolution by an equivalent random evolution of pure states is known as the quantum trajectory approach to open system dynamics, and is also called unraveling of the master equation [18]. The advantages of the description in terms of random pure states over the description by density matrix $\hat{\rho}$ are twofold. The computations are much more practical, as soon as the size of the Hilbert space is moderate or large [18]. On the theoretical side, the stochastic evolution of pure states provides valuable insights which cannot be inferred from the density matrix approach [17-21]. Non-uniqueness of the representation of the mixed state in terms of pure states implies that there are several different types of unraveling which provide different insights into the dynamics of the open system.

We shall exploit the fact that QSD equation is the unique unraveling of (1) which has the same invariance as (1) under the unitary transformations of the environment operators [17]. The linear form of QSD equation is given by the following formula:

$$
\begin{aligned}
& |\mathrm{d} \varphi\rangle=\left[-\mathrm{i} \hat{H} \mathrm{~d} t-\sum_{m} \hat{L}_{m}^{\dagger} \hat{L}_{m} \mathrm{~d} t+\sum_{m} \hat{L}_{m} d w_{m}\right] \\
& \quad \times|\varphi(t)\rangle,
\end{aligned}
$$

where $w_{m}$ are complex Wiener processes satisfying

$$
\begin{aligned}
& E_{Q}\left[\mathrm{~d} w_{m}\right]=E_{Q}\left[\mathrm{~d} w_{m} \mathrm{~d} w_{m^{\prime}}\right]=0, \\
& E_{Q}\left[\mathrm{~d} w_{m} \mathrm{~d} w_{m^{\prime}}^{*}\right]=2 \delta_{m, m^{\prime}} \mathrm{d} t .
\end{aligned}
$$

$E_{Q}[\cdot]$ denotes the expectation with respect to the stochastic process. The stochastic process with increment $|\mathrm{d} \varphi\rangle$ given by (3) satisfies the unraveling property

$$
\operatorname{Tr}[\hat{\rho}(t) \hat{A}]=E_{Q}[\langle\varphi(t)|\hat{A}| \varphi(t)\rangle],
$$

for any operator $\hat{A}$ and for all times $t$. Equation (3) is linear and does not preserve the norm of wave function. There exists a nonlinear norm preserving form of QSD equation which is more convenient for efficient simulations of open system dynamics. However, we shall use, in a crucial way, the linear form of the QSD theory.

It can be easily seen that the stochastic process is invariant under the transformation. In fact, the substitution of (2) leads to the same equation with $\mathrm{d} w_{m}^{\prime}=$ $\sum_{k} u_{k m} \mathrm{~d} w_{k}$ and $\mathrm{d} w_{m}^{*^{\prime}}=\sum_{k} u_{k m}^{*} \mathrm{~d} w_{k}^{*}$ instead of $\mathrm{d} w_{m}$ and $\mathrm{d} w_{m}^{*}$, but these have the same stochastic properties.
Let us consider an orbit $\hat{\rho}(t), t \in\left(t_{0}, t_{1}\right)$ of (1). The initial mixed state is a convex combination of pure state projectors $\hat{\rho}\left(t_{0}\right)=\sum_{k} p_{k}\left|\varphi_{0}^{k}\right\rangle\left\langle\varphi_{0}^{k}\right|$. Starting from initial condition $\varphi_{w}^{k}\left(t_{0}\right)=\varphi_{0}^{k}$ each of the pure states is evolved stochastically through the space of pure states using Eq. (3) resulting in $\left|\varphi_{w}^{k}(t)\right\rangle$, where subscript $w$ corresponds to the sample paths of (3). Deterministic evolution through mixed states by (1) from the initial pure state $\left|\varphi_{0}^{k}\right\rangle\left\langle\varphi_{0}^{k}\right|$ gives the curve $\hat{\rho}^{k}(t)$ which is equal to $E_{Q}\left[\left|\varphi_{w}^{k}(t)\right\rangle\left\langle\varphi_{w}^{k}(t)\right|\right], t \in\left(t_{0}, t_{1}\right)$. Physical motivation based on the interferometric approach, elaborated in [16], requires the total phase to be defined using the linear QSD equation as follows:

$$
\alpha_{\text {tot }}^{k}(t)=\arg E_{Q}\left[\left\langle\varphi_{w}^{k}\left(t_{0}\right) \mid \varphi_{w}^{k}(t)\right\rangle\right] .
$$

The dynamical phase related to the curve $\hat{\rho}^{k}(t)$ can be defined as

$$
\alpha_{\mathrm{dyn}}^{k}(t)=\operatorname{Im} \int_{t_{0}}^{t} E_{Q}\left[\left\langle\varphi_{w}^{k}(s) \mid \mathrm{d} \varphi_{w}^{k}(s)\right\rangle\right] .
$$

The phases (6) and (7) are well defined and uniquely associated with the curve of mixed states $\hat{\rho}^{k}(t)$, as will be presently demonstrated and illustrated with examples. Finally, the geometric phase of the curve $\hat{\rho}^{k}(t)$ is uniquely defined as the difference of the total and the dynamical phases

$$
\alpha_{\mathrm{g}}^{k}(t)=\alpha_{\mathrm{tot}}^{k}(t)-\alpha_{\mathrm{dyn}}^{k}(t) .
$$

This completes the definition of the phases in the case of pure initial states.

Let us briefly demonstrate that the phases (6) and (7) are invariant under the transformations. The expression $\langle\varphi \mid \mathrm{d} \varphi\rangle$ after substitution of (2) into (3) and using the properties of $u_{m k}$ becomes

$$
\begin{aligned}
& \langle\varphi \mid \mathrm{d} \varphi\rangle=-\mathrm{i}\langle\hat{H}\rangle_{\varphi} \mathrm{d} t-\sum_{k}\left\langle\hat{L}_{k}^{\dagger} \hat{L}_{k}\right\rangle_{\varphi} \mathrm{d} t \\
& \quad+\sum_{k}\left\langle\hat{L}_{k}\right\rangle_{\varphi} \mathrm{d} w_{k}^{\prime},
\end{aligned}
$$

where $\langle\cdot\rangle_{\varphi}$ denotes the quantum expectation in the state $|\varphi(t)\rangle$. Notice that, due to the unitarity of $u_{m k}$, the stochastic increments $\mathrm{d} w_{k}^{\prime}$ in (9) satisfy the same properties as $\mathrm{d} w_{k}$ and thus generate the same stochastic process $\left|\varphi_{w}^{k}(t)\right\rangle$. From the invariance of $\langle\varphi \mid \mathrm{d} \varphi\rangle$ follows the invariance of the dynamical phase along an orbit $\hat{\rho}^{k}(t)$ of (1). In a similar manner, it is obvious that the total phase is also invariant because the same stochastic process $\left|\varphi_{w}^{k}(t)\right\rangle$ is generated. Furthermore, the imaginary part of $(7)$ is always equal to $-\langle\hat{H}\rangle_{\varphi} \mathrm{d} t$. Using the unraveling property (5) we get simple expression of dynamical phase

$$
\alpha_{\mathrm{dyn}}^{k}(t)=-\int_{t_{0}}^{t} \operatorname{Tr}\left[\hat{\rho}^{k}(s) \hat{H}\right] \mathrm{d} s .
$$

Let us note that the dynamical phase depends on the environment only through the evolution of the state. Thus, the geometric phase is well defined and uniquely associated with the orbit $\hat{\rho}^{k}(t)$ of the LME.

\section{Acknowledgments}

This work is partly supported by the Serbian Ministry of Science contract No. 141003. 


\section{References}

[1] M.V. Berry, Proc. R. Soc. London, Ser. A 392, 45 (1984).

[2] B. Simon, Phys. Rev. Lett. 51, 2167 (1983).

[3] Geometric Phases in Physics, Eds. A. Shapere, F. Wilczek, World Sci., Singapore 1989.

[4] Y. Aharonov, J. Anandan, Phys. Rev. Lett. 58, 1593 (1987).

[5] J. Samuel, R. Bhandari, Phys. Rev. Lett. 60, 2339 (1988).

[6] A.K. Pati, Phys. Rev. A 52, 2576 (1995).

[7] N. Mukunda, R. Simon, Ann. Phys. (N.Y.) 228, 205 (1993).

[8] P. Zanardi, M. Rosetti, Phys. Lett. A 264, 94 (1999).

[9] A. Uhlmann, Rep. Math. Phys. 24, 229 (1986).

[10] E. Sjöqvist, A.K. Pati, A. Ekert, J.S. Anandan, M. Ericsson, D.K.L. Oi, V. Vedral, Phys. Rev. Lett. 85, 2845 (2000).

[11] M. Ericsson, E. Sjöqvist, J. Brannlund, D.K.L. Oi, A.K. Pati, Phys. Rev. A 67, 020101(R) (2003).
[12] D.M. Tong, E. Sjöqvist, L.C. Kwek, C.H. Oh, Phys. Rev. Lett. 93, 080405 (2004).

[13] A. Carollo, I. Fuentes-Guridi, M.F. Santos, V. Vedral, Phys. Rev. Lett. 90, 160402 (2003).

[14] M.S. Sarandy, D.A. Lidar, Phys. Rev. A 73, 062101 (2006).

[15] H. Goto, K. Ichimura, Phys. Rev. A 76, 012120 (2007).

[16] A. Bassi, E. Ippoliti, Phys. Rev. A 73, 062104 (2006).

[17] I.C. Percival, Quantum State Diffusion, Cambridge University Press, Cambridge 1999.

[18] H.-P. Breuer, F. Petruccione, The Theory of Open Quantum Systems, Oxford University Press, Oxford 2001.

[19] N. Buric, Phys. Rev. A 73, 052111 (2006).

[20] N. Buric, Phys. Rev. A 77, 012321 (2008).

[21] N. Buric, Phys. Rev. A 79, 022101 (2009). 